\title{
Screening of the residual normal ovarian tissue adjacent to orthotopic epithelial ovarian carcinomas in nude mice
}

\author{
G.H. Zhu' ${ }^{1}$, S.T. Wang ${ }^{1}$, M.Z. Yao ${ }^{1}$, J.H. Cai ${ }^{3}$, C.Y. Chen ${ }^{1}$, Z.X. Yang ${ }^{2}$, \\ L. Hong ${ }^{1}$ and S.Y. Yang ${ }^{1}$ \\ ${ }^{1}$ Department of Gynecology, Hainan Provincial People's Hospital, Haikou, \\ Hainan, China \\ ${ }^{2}$ Hainan Medical College, Drug Safety Evaluation Center, Haikou, Hainan, \\ China \\ ${ }^{3}$ Research Center, Hainan Provincial People's Hospital, Haikou, Hainan, China \\ Corresponding author: G.H. Zhu \\ E-mail: genhaizhu@163.com
}

Genet. Mol. Res. 13 (2): 2978-2986 (2014)

Received April 8, 2013

Accepted October 31, 2013

Published April 16, 2014

DOI http://dx.doi.org/10.4238/2014.April.16.6

\begin{abstract}
The objective of this study was to explore the feasibility and methods of screening the residual normal ovarian tissue adjacent to orthotopic ovarian carcinomas in nude mice. Human epithelial ovarian cancer cells (OVCAR3) were subcutaneously implanted for a tumor source and ovarian orthotopic transplantation. The cancer tissue, proximal paraneoplastic tissue, middle paraneoplastic tissue, remote paraneoplastic tissue, and normal ovarian tissue were removed. CK-7, CA125, p53, survivin, MMP-2, and TIMP-2 expression was detected by reverse transcription polymerase chain reaction. We obtained 35 paraneoplastic residual ovarian tissues with normal biopsies from 40 cases of an orthotopic epithelial ovarian carcinoma model (87.5\%). CK-7, CA125, p53, survivin, MMP-2, and TIMP-2 expression was lower in proximal paraneoplastic tissue than in cancer tissue $(\mathrm{P}<0.05)$ and higher than in middle and remote paraneoplastic tissue $(\mathrm{P}<0.01)$.
\end{abstract}


There was no statistically significant difference between the expression of these genes in middle and proximal paraneoplastic tissue as well as among residual normal ovarian tissues with different severity $(\mathrm{P}>$ 0.05 ). In ovarian tissues of 20 normal nude mice, the expression of CK7, CA125, p53, survivin, MMP-2, and TIMP-2 was negative. Overall, the expression levels of CK-7, CA125, p53, survivin, MMP-2, TIMP-2, and other molecular markers showed a decreasing trend in the noncancer tissue direction. The expression levels can be used as standards to screen residual normal ovarian tissue. We can obtain relatively safe normal ovarian tissues adjacent to epithelial ovarian cancer.

Key words: Epithelial ovarian cancer; Tumor-supplied group of factors; Gene expression; Paraneoplastic tissue

\section{INTRODUCTION}

The treatment of ovarian cancer mainly includes surgery and chemotherapy. Compared with other types of ovarian malignant tumors, epithelial ovarian cancer patients have strict conditions to retain ovarian function and reproductive function, which is only suitable for young and early-stage patients (Kajiyama et al., 2011). Most of the patients undergo a comprehensive staging surgery, and this means the permanent loss of ovarian function after the operation. A considerable portion of women with epithelial ovarian cancer are in their childbearing age. The absence of ovarian function will lead to early menopause, with symptoms such as hot flashes, insomnia, body aches, irritability, more serious genitourinary tract infections, and earlier appearance of related diseases such as osteoporosis, coronary heart disease, and metabolic syndrome (Michelsen et al., 2009). Besides, the quality of life of patients is compromised (Finch and Narod, 2011). At present, hormone replacement therapy (HRT) is used for treatment. Because of the concerns of doctors and patients regarding HRT, research about patients with epithelial ovarian cancer using HRT after surgery is rare. No staple clinic research has systematically certified the safety of the treatment. However, the obvious side effects and adverse consequences of HRT are definite (MacLennan, 2011). Additionally, its clinic application is restricted. Therefore, it is necessary to find a more secure and effective method to solve the problem of ovarian function loss after surgery.

In recent years, many scholars tried to transplant freeze-thawed ovarian tissue to restore ovarian function in patients with leukemia, breast cancer, cervical cancer, Hodgkin's lymphoma, colon cancer, and osteosarcoma (Donnez et al., 2006; Georgescu et al., 2008; Rosendahl et al., 2008). Although these studies lack long-term follow-up results and risk assessment, they can, at least, indicate that transplanting freeze-thawed ovarian tissue is an effective method to restore ovarian endocrine function in some patients with malignancy. Thus, we can suppose that freeze-thawed ovarian tissue transplantation can be used to restore ovarian endocrine function in patients with epithelial ovarian cancer. We searched the literature, but we did not find clinical and experimental studies on freeze-thawed ovarian tissue transplantation to patients with epithelial ovarian cancer after surgery. This may be because of the characteristics of epithelial ovarian cancer that make it different from other malignancies, that transplantation of epithelial ovarian cancer patients' own ovarian tissue has a high risk of reimplanting cancer. 
In order to solve the cancer cell re-implantation problem, some researchers used ovary cells and primordial follicles for cell culture. Although some success was reported, the rate is low. This method is mostly used for in vitro fertilization and is not suitable for recovering ovarian endocrine function after surgery for malignancy. Theoretically, the cancer re-implantation problem can also be solved by marking specific genes of ovarian cancer cells. However, ovarian cancer has polygenic regulation, and a specific gene has not been identified. Whether there is a relatively safe method to circumvent the re-implantation of residual tumor cells or subclinical metastatic cells remains unknown. In order to avoid the re-implantation of residual cancer cells, it is very important to screen and secure normal ovarian tissue for transplantation. Recently, reverse transcription polymerase chain reaction (RT-PCR) was found to be an effective method for screening residual focal cancer, micrometastasis, and hidden metastatic carcinoma (Lioznov et al., 2008). There are numerous genes that are related to ovarian cancer, making it difficult to choose marker genes. According to Meirow's report, the expression of CD30, T cell receptor, and BCR-ABI can be used to check residual micro focus in transplanted ovarian tissue of patients with Hodgkin disease, lymphoma, and leukemia, respectively (Meirow et al., 2008). Genes related to these diseases are expressed at levels that are appropriate for monitoring. We can screen related genes that mediate the corrosion and metastasis of ovarian cancer. There is a series of genes that includes cytokeratin-7 (CK-1), CA125, matrix metalloproteinase-2 (MMP-2), tissue inhibitor of metalloproteinase-2 (TIMP-2), survivin, and p53. They are frequently used in clinical applications, and their function of mediating ovarian cancer corrosion and metastasis has been confirmed (Takami et al., 2012). Therefore, they are suitable to use as relatively ideal molecular markers to screen residual cancer cells or forecast hidden metastatic carcinoma. We aimed to screen relatively secure normal ovarian tissue for transplant by detecting the above molecular markers in tissues that were adjacent to orthotopic human epithelial ovarian carcinomas in nude mice.

\section{MATERIAL AND METHODS}

\section{Materials}

The human epithelial ovarian cancer cell line OVCAR3 was purchased from American Type Culture Collection (ATCC, Manassas, VA, USA). Fetal bovine serum and RPMI1640 medium were purchased from Invitrogen (Carlsbad, CA, USA).

Female BALB/c nude mice, which were 4-6 weeks old and weighed 15-17 g, were purchased from the Experimental Animal Center of Guangdong Province. Mice were fed within the special pathogen-free (SPF) class barrier system. Feed and bedding materials were all sterilized. Also, people who went into the laboratory and all goods were subjected to strict microbial control.

Total RNA extraction reagent (the RNeasy Minikit kit) was purchased from QIAGEN. RT-PCR reagents, primers, and internal references were bought from Shanghai Sangon Biological Engineering Co., Ltd.

\section{Cell culture}

Medium was added after cell recovery, and the cells were centrifuged at a low speed. The 
supernatant was removed, and the cells were subcultured at $37^{\circ} \mathrm{C}$ and $5 \% \mathrm{CO}_{2}$ in RPMI-1640 medium containing $10 \%$ new bovine serum, $200 \mu / \mathrm{mL}$ penicillin, and $200 \mu / \mathrm{mL}$ streptomycin.

\section{Establishment of the orthotopic epithelial ovarian carcinoma model}

Logarithmic-phase OVCAR3 cells were treated with $0.25 \%$ trypsin, and cells were suspended in serum-free medium. Cells were centrifuged and resuspended in phosphate-buffered saline (PBS) at a density of $2 \times 10^{7}$ cells $/ \mathrm{mL}$. Ten nude mice received $0.2 \mathrm{~mL}$ cell suspension by subcutaneous injection in the armpit near the neck or back. The tumor formation rate was $90 \%$ after $4-8$ weeks, and tumors were examined by pathological biopsy. Under sterile conditions, a subcutaneous tumor source was trimmed to be $1 \times 1 \times 1 \mathrm{~mm}$ tissue blocks. To establish the orthotopic transplantation tumor model, mice received intraperitoneal anesthesia by $1 \%$ amyl barbital sodium $(45 \mathrm{mg} / \mathrm{kg}$ body weight). A small tumor block was inoculated into the left ovarian capsule under the microscope and instilled with OB glue on the surface, which was in the abdominal cavity after solidification. Then, we sutured the subcutaneous layer and skin using No. 0 silk. Forty-five nude mice were inoculated, and 40 developed tumors from the orthotopic implantation, yielding a success rate of $88.9 \%$.

\section{Anatomy}

We dissected 10 nude mice every 2 weeks after orthotopic implantation for 4 weeks, recording tumor size, bilateral ovarian involvement, ascites, abdominopelvic cavity planting degree, and metastasis of the aortic lymph node. We removed the ovary with the tumor and equidistantly cut the adjacent tissues into 3 parts, the proximal, middle, and remote paraneoplastic tissue. Each section was cut into 1-mm sheets of tissue, which were used for tissue biopsy. Ovarian tissues with normal biopsy were conserved in liquid nitrogen for further testing. At the same time, 20 nude mice underwent the same procedure as a control, but they received an orthotopic transplantation of ovarian tissue from normal nude mice.

\section{Semi-quantitative RT-PCR}

The frozen tissue was ground into powder in liquid nitrogen. Then, the specified buffer was added, the supernatant was centrifuged, and the total RNA was extracted according to the RNeasyMinikit manual. We eliminated residual DNA by Dnase digestion and detected the concentration and purity of RNA using an ultraviolet spectrometer. To detect any DNA contamination, PCR amplification was performed using $\beta$-actin primers. Ultraviolet light was used to detected 50 ng RNA that was electrophoresed on 1\% agarose gels. According to the kit manual, $4 \mu \mathrm{g}$ RNA was reversed transcribed into cDNA with Supercript II, and the semiquantitative PCR reaction system was $25 \mu \mathrm{L}$. Primers of CK-7, CA125, MMP-2, TIMP-2, survivin, and $\mathrm{p} 53$ are listed in Table 1.

The following PCR conditions were used. Survivin: $94^{\circ} \mathrm{C}$ denaturation for $1 \mathrm{~min}$, $56^{\circ} \mathrm{C}$ annealing for $1 \mathrm{~min}$, and $72^{\circ} \mathrm{C}$ extension for $2 \mathrm{~min}$; MMP-2: $94^{\circ} \mathrm{C}$ denaturation for 1 min, $58^{\circ} \mathrm{C}$ annealing for $1 \mathrm{~min}$, and $72^{\circ} \mathrm{C}$ extension for $2 \mathrm{~min}$; TIMP-2: $94^{\circ} \mathrm{C}$ denaturation for $1 \mathrm{~min}, 58^{\circ} \mathrm{C}$ annealing for $2 \mathrm{~min}$, and $72^{\circ} \mathrm{C}$ extension for $2 \mathrm{~min}$; CK-7: $94^{\circ} \mathrm{C}$ denaturation for $30 \mathrm{~s}, 55^{\circ} \mathrm{C}$ annealing for $30 \mathrm{~s}$, and $72^{\circ} \mathrm{C}$ extension for $30 \mathrm{~s}$; and $\mathrm{p} 53: 94^{\circ} \mathrm{C}$ denaturation for 
$30 \mathrm{~s}, 50^{\circ} \mathrm{C}$ annealing for $30 \mathrm{~s}$, and $72^{\circ} \mathrm{C}$ extension for $2.5 \mathrm{~min}$. All reactions had a final extension at $72^{\circ} \mathrm{C}$ for 5 min after 35 cycles, and the products were stained by ethidium bromide. Automated gel imaging analyzed the result of $2 \%$ agarose gel electrophoresis to detect the integral optical density (integrated optical densities, IODs). Then, we calculated the IOD ratio of experimental and reference genes.

Table 1. Primer sequences of CK-7, CA125, MMP2/TIMP-2, survivin, and P53.

\begin{tabular}{llll}
\hline Primers & Positive sense strand & Antisense strand & Length (bp) \\
\hline$\beta$-actin & AAGCAGGAGTATGACGAGTCCG & GCCTTCATACATCTCAAGTTGG & 559 \\
Survivin & CCGCAACTGAGATTGTGGGAAT & GGATCATCTGCCTGAGTATAAT & 301 \\
MMP2 & CCTGTCTTGCGTGCATAGGT & CGATTCTGCCCTCCTCCTTCG & 102 \\
TIMP-2 & TGGCAGACTACAATACAAGAGC & AGATGTGGTAGGAGATGATGGG & 140 \\
CA125 & CCCCTACACTGGCGAAGA & GGTGGCTTCTGAAGTGGC & 101 \\
CK-7 & GTTCCATTTGCAAAGGCTGT & CAGGTGGTTATCCCGAAAGA & 292 \\
P53 & AGGACCCTGTCACCGAGA & GTGGAAGCCATAGTTGCC & 125 \\
\hline
\end{tabular}

\section{Statistical analysis}

We compared the mean differences by the Student $t$-test and the rates by the chisquare test. Statistical analysis was conducted by the SPSS 13.0 software, and P $<0.05$ was considered to be statistically significant.

\section{RESULTS}

\section{Extent of xenograft ovarian tumor lesions in situ and the acquisition rate of residual normal ovarian tissue}

Ovarian cancer tumors were observed 2 weeks after transplantation, and widespread metastasis was observed after 8 weeks; metastasis sites included the liver, intestines, peritoneum, lymph nodes, other parts of the transfer, and ascites. Tumors were detected in $87.5 \%$ $(35 / 40)$ of normal ovarian tissues by pathological examination. A potential for tumor transfer may exist in these tissues, and they should be screened further (Table 2).

Table 2. Involving extent of xenograft ovarian tumor lesions in situ and the acquisition rate of residual normal ovarian tissue.

\begin{tabular}{|c|c|c|c|c|}
\hline & 2 Weeks & 4 Weeks & 6 Weeks & 8 Weeks \\
\hline Anatomy No. & 10 & 10 & 10 & 10 \\
\hline Only ovarian planting & $10(10)$ & $4(4)$ & $1(1)$ & 0 \\
\hline Hepatic metastases & 0 & $2(2)$ & $2(2)$ & $2(2)$ \\
\hline Intestinal metastases & 0 & $2(2)$ & $3(3)$ & $1(1)$ \\
\hline Ascites & 0 & $2(1)$ & $2(1)$ & $3(2)$ \\
\hline Retroperitoneal lymph node metastasis & 0 & 0 & $1(1)$ & $2(1)$ \\
\hline Widespread metastasis & 0 & 0 & $1(1)$ & $2(1)$ \\
\hline The acquisition rate of residual normal ovary tissue (\%) & $10(100 \%)$ & $9(90 \%)$ & $9(90 \%)$ & $7(70 \%)$ \\
\hline
\end{tabular}

mRNA expression of CK-7, CA125, p53, survivin, MMP-2, and TIMP-2

The mRNA expression of CK-7, CA125, p53, survivin, MMP-2, and TIMP-2 was de- 
tected in remote paraneoplastic tissues, middle paraneoplastic tissues, proximal paraneoplastic tissues, and cancer tissue by electrophoresis of RT-PCR products (Figure 1). Furthermore, the expression of CK-7, p53, CA125, survivin, MMP-2, and TIMP-2 was detected at different levels in cancer tissue and paraneoplastic tissues that were different distances from the tumor source, while the expression of these genes was not be detected in normal ovarian tissue of nude mice. The expression of CK-7, CA125, p53, survivin, MMP-2, and TIMP-2A was not detected in paraneoplastic residual normal ovarian tissue from 21 patients (Table 3 ).
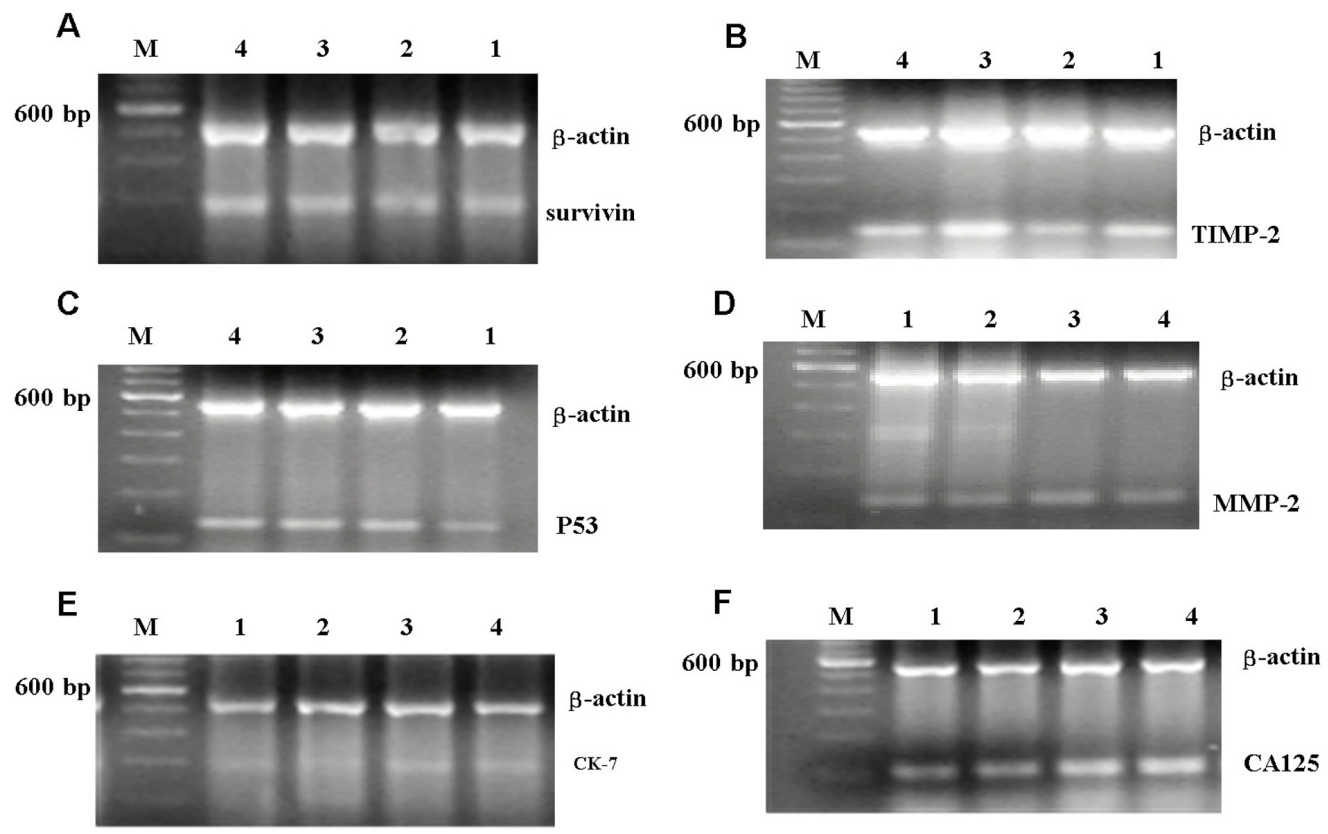

Figure 1. RT-PCR electrophoresis result of CK-7, CA125, p53, survivin, MMP-2, TIMP-2. Lane M = marker; lane 1 = remote paraneoplastic tissues; lane 2 = middle paraneoplastic tissues; lane $3=$ proximal paraneoplastic tissues; lane $4=$ cancer tissue.

Table 3. Positive rate detected by RT-PCR of different molecular markers in cancer tissue and paraneoplastic tissues with different distance (cases \%).

\begin{tabular}{lccccccc}
\hline & Cases & CK-7 & CA125 & P53 & Survivin & MMP-2 & TIMP-2 \\
\hline Cancer tissue & 40 & $39(97.5)$ & $39(97.5)$ & $36(90.0)$ & $38(95.0)$ & $38(95.0)$ & $38(95.0)$ \\
Paraneoplastic tissues & - & - & - & - & - & - & - \\
Proximal paraneoplastic & 35 & $26(74.3)^{\mathrm{a}}$ & $27(77.1)^{\mathrm{a}}$ & $23(65.7)^{\mathrm{a}}$ & $25(71.4)$ & $24(68.6)^{\mathrm{a}}$ & $26(74.3)^{\mathrm{a}}$ \\
Middle paraneoplastic & 35 & $14(40.0)^{\mathrm{b}}$ & $12(34.3)^{\mathrm{b}}$ & $11(31.4)^{\mathrm{b}}$ & $12(34.3)^{\mathrm{b}}$ & $13(37.1)^{\mathrm{b}}$ & $13(37.1)^{\mathrm{b}}$ \\
Remote paraneoplastic & 35 & $13(37.1)^{\mathrm{c}}$ & $11(31.4)^{\mathrm{c}}$ & $11(31.4)^{\mathrm{c}}$ & $10(28.6)$ & $12(34.3)^{\mathrm{c}}$ & $11(31.4)^{\mathrm{c}}$ \\
\hline
\end{tabular}

${ }^{\mathrm{a} C o m p a r e d}$ with cancer tissue, $\mathrm{P}<0.05$; ${ }^{\mathrm{b}}$ campared with proximal paraneoplastic, $\mathrm{P}<0.01$; ${ }^{\mathrm{c}}$ campared with middle paraneoplastic, $\mathrm{P}>0.05$.

The expression of CK-7, CA125, P53, survivin, MMP-2, and TIMP-2 was lower in proximal paraneoplastic tissue than in cancer tissue $(\mathrm{P}<0.05)$ and higher in proximal paraneoplastic tissue than in middle and remote paraneoplastic tissue $(\mathrm{P}<0.01)$. There was no 
statistically significant difference between the expression levels in middle and proximal paraneoplastic tissue as well as among residual normal ovarian tissues with different severity $(\mathrm{P}>$ 0.05) (Table 4).

Table 4. RT-PCR detection of ovarian tissue molecular indexes in cancer tissue and paraneoplastic tissues with different distance and normal biopsy.

\begin{tabular}{lcccccc}
\hline & Cases & CK-7 & CA125 & P53 & Survivin & MMP-2/TIMP-2 \\
\hline Cancer tissue & 40 & $1.94 \pm 0.26$ & $1.97 \pm 0.22$ & $1.93 \pm 0.29$ & $1.98 \pm 0.26$ & $1.95 \pm 0.25$ \\
Paraneoplastic tissues & - & - & - & - & - \\
Proximal paraneoplastic & 35 & $1.15 \pm 0.11^{\mathrm{a}}$ & $1.24 \pm 0.13^{\mathrm{a}}$ & $1.65 \pm 0.31^{\mathrm{a}}$ & $1.39 \pm 0.15^{\mathrm{a}}$ & $1.14 \pm 0.12^{\mathrm{a}}$ \\
Middle paraneoplastic & 35 & $0.67 \pm 0.05^{\mathrm{b}}$ & $0.82 \pm 0.08^{\mathrm{b}}$ & $1.12 \pm 0.17^{\mathrm{b}}$ & $0.87 \pm 0.07^{\mathrm{b}}$ & $0.78 \pm 0.06^{\mathrm{b}}$ \\
Remote paraneoplastic & 35 & $0.69 \pm 0.05^{\mathrm{c}}$ & $0.79 \pm 0.07^{\mathrm{c}}$ & $0.96 \pm 0.08^{\mathrm{c}}$ & $0.91 \pm 0.08^{\mathrm{c}}$ & $0.71 \pm 0.07^{\mathrm{c}}$ \\
\hline
\end{tabular}

${ }^{a}$ Compared with cancer tissue, $\mathrm{P}<0.05$; ${ }^{\mathrm{b}}$ campared with proximal paraneoplastic, $\mathrm{P}<0.01$; ${ }^{\mathrm{c}}$ campared with middle paraneoplastic, $\mathrm{P}>0.05$.

\section{RT-PCR detection of molecular markers of xenograft residual normal ovarian tissue}

We obtained 15 cases of residual normal ovarian tissue only by ovarian planting and 20 cases by ovarian outside planting, including liver metastases, intestinal metastasis, ascites, retroperitoneal lymph node metastasis, and extensive transfer. The expression of CK-7, CA125, p53, survivin, MMP-2, TIMP-2, and other molecular markers were not different in these 2 groups (Table 5).

Table 5. RT-PCR detection of molecular markers of residual normal ovarian with different lesions degrees (means \pm SD).

\begin{tabular}{lccccccc}
\hline & Cases & CK-7 & CA125 & P53 & Survivin & MMP-2/TIMP-2 \\
\hline Only ovarian planting & 15 & $0.65 \pm 0.05$ & $0.86 \pm 0.09$ & $1.92 \pm 0.30$ & $0.89 \pm 0.08$ & $0.76 \pm 0.07$ \\
Ovarian outside planting & 20 & $0.72 \pm 0.05$ & $0.88 \pm 0.07$ & $1.95 \pm 0.28$ & $0.93 \pm 0.07$ & $0.80 \pm 0.06$ \\
\hline
\end{tabular}

Ovarian planting compared with ovarian outside planting, $\mathrm{P}>0.05$.

\section{DISCUSSION}

Autologous transplantation of ovarian tissue has been used to restore ovarian function in various malignant tumor patients (Gracia et al., 2012), but its use is limited in epithelial ovarian cancer patients because of the risk of re-implanting residual cancer cells or potential malignant cells. Therefore, the key is to obtain relatively secure normal ovarian tissue to avoid re-implanting residual tumor cells or cells with a subclinical metastasis tendency. RT-PCR proves the existence of tumor cells by amplifying mRNA in tumor cells, and it is considered to be a specific method with high sensitivity to detect residual tumor cells or hidden metastasis (Jennings et al., 2012). CK-7 is expressed stably in primary ovarian cancer cells or metastases from ovary and is a common marker to determine whether the metastases came from ovary (Heatley, 2008). As a common cancer indicator for epithelial ovarian cancer, CA125 is highly expressed in ovarian cancer tissue. CA125 is frequently used to identify residual metastases after chemotherapy for ovarian cancer and to further identify negative tissue in common disease inspection (Miller et al., 2008). Nearly all epithelial ovarian cancers with a high degree of malignancy express mutant p53 (Romero and Bast, 2012), which is a target gene of epithelial ovarian cancer treatment 
(Rahma et al., 2012). The survivin gene has little or low expression in normal tissues and is a common molecular marker for monitoring cancer metastasis and judging the effectiveness of treatment and prognosis (Aune et al., 2011; Felisiak-Golabek et al., 2011). Because adhesion and extracellular matrix degradation is the first step in ovarian cancer invasion and metastasis, MMP-2/TIMP-2 is expressed highly in epithelial ovarian cancer tissues. Unlike other members of the MMP/TIMP family, MMP-2/TIMP-2 has low expression in benign ovarian tumor tissues (Brun et al., 2008). These genes are suitable to be molecular markers for screening residual focal cancer and forecasting hidden metastasis. We showed that a transition area, which appears to be normal in common pathology examination, exists in tissues adjacent to tumors. The expression of molecular markers, including CK-7, CA125, p53, survivin, MMP-2, and TIMP-2, show a decreased expression tendency in the no-cancer direction. The expression of CK-7, CA125, p53, survivin, MMP-2, and TIMP-2 in residual normal ovarian tissues that are adjacent to focal cancer is close to that in cancer tissues, while the expression of these molecular markers in residual normal ovarian tissues that are far away from focal cancer is low. Though the residual normal ovarian tissues that are adjacent to focal cancer appear normal by pathologic biopsy, the expression of CK-7, CA125, p53, survivin, MMP-2, and TIMP-2 is dramatically different. Tissues is this transition area have a high risk of hidden metastasis or micro invasion. This suggests that common pathology examination cannot be used to exclude metastatic potential. However, molecular markers, including CK-7, CA125, p53, survivin, MMP-2, and TIMP-2, may be used to help to screen relatively secure ovarian tissues. This also indicates that normal ovarian tissue can be found in pathology examination even in widespread metastasis. Molecular markers in residual normal ovarian tissue do not change much in the case of ascites, which suggests that there is still a chance that normal ovarian tissues can be acquired for transplantation even in the case of widespread metastasis. Actually, it was reported that even patients with end-stage ovarian cancer have had successful operations in which the focal cancer was removed and the residual tissues functioned well without metastasis (Miller et al., 1997; Dykgraaf et al., 2009). As long as we regulate screening conditions, residual normal ovarian tissues can be transplanted securely. No standard for screening ovarian tissues by RT-PCR has been reported yet. Because of the low or no expression of genes, such as CK-7, CA125, p53, survivin, MMP-2, and TIMP-2, in normal ovarian tissues, the expression of these markers is defined as negative in the screening standard. With this standard, 21 normal ovarian tissues were screened and cryopreserved.

Among the methods that are currently used to screen residual cancer cells, RT-PCR has some advantages, but there are also some flaws. The most notable flaws of this method are false-positive results that are caused by the amplification of contaminants and false-negative results that result from poor control of experimental conditions. Also, RT-PCR cannot be used to determine the morphology of a tumor cell. Whether the 21 cases of normal ovarian tissue that were screened by the above molecular markers are safe remains to be further examined by transplanting them back into nude mice.

\section{ACKNOWLEDGMENTS}

Research supported by the National Natural Science Foundation (\#30960408).

\section{REFERENCES}

Aune G, Stunes AK, Tingulstad S, Salvesen O, et al. (2011). The proliferation markers Ki-67/MIB-1, phosphohistone H3, and survivin may contribute in the identification of aggressive ovarian carcinomas. Int. J. Clin. Exp. Pathol. 4: 444-453. 
Brun JL, Cortez A, Commo F, Uzan S, et al. (2008). Serous and mucinous ovarian tumors express different profiles of MMP-2, -7, -9, MT1-MMP, and TIMP-1 and -2. Int. J. Oncol. 33: 1239-1246.

Donnez J, Dolmans MM, Demylle D, Jadoul P, et al. (2006). Restoration of ovarian function after orthotopic (intraovarian and periovarian) transplantation of cryopreserved ovarian tissue in a woman treated by bone marrow transplantation for sickle cell anaemia: case report. Hum. Reprod. 21: 183-188.

Dykgraaf RH, de Jong D, van Veen M, Ewing-Graham PC, et al. (2009). Clinical management of ovarian small-cell carcinoma of the hypercalcemic type: a proposal for conservative surgery in an advanced stage of disease. Int. J. Gynecol. Cancer 19: 348-353.

Felisiak-Golabek A, Rembiszewska A, Rzepecka IK, Szafron L, et al. (2011). Nuclear survivin expression is a positive prognostic factor in taxane-platinum-treated ovarian cancer patients. J. Ovarian Res. 4: 20.

Finch A and Narod SA (2011). Quality of life and health status after prophylactic salpingo-oophorectomy in women who carry a BRCA mutation: A review. Maturitas 70: 261-265.

Georgescu ES, Goldberg JM, du Plessis SS and Agarwal A (2008). Present and future fertility preservation strategies for female cancer patients. Obstet. Gynecol. Surv. 63: 725-732.

Gracia CR, Chang J, Kondapalli L, Prewitt M, et al. (2012). Ovarian tissue cryopreservation for fertility preservation in cancer patients: successful establishment and feasibility of a multidisciplinary collaboration. J. Assist. Reprod. Genet. 29: 495-502.

Heatley MK (2008). Immunohistochemical biomarkers of value in distinguishing primary ovarian carcinoma from gastric carcinoma: a systematic review with statistical meta-analysis. Histopathology 52: 267-276.

Jennings LJ, Smith FA, Halling KC, Persons DL, et al. (2012). Design and analytic validation of BCR-ABL1 quantitative reverse transcription polymerase chain reaction assay for monitoring minimal residual disease. Arch. Pathol. Lab. Med. 136: 33-40.

Kajiyama H, Shibata K, Mizuno M, Umezu T, et al. (2011). Long-term survival of young women receiving fertility-sparing surgery for ovarian cancer in comparison with those undergoing radical surgery. Br. J. Cancer 105: 1288-1294.

Lioznov M, Badbaran A, Fehse B, Bacher U, et al. (2008). Monitoring of minimal residual disease in multiple myeloma after allo-SCT: flow cytometry vs PCR-based techniques. Bone Marrow Transplant. 41: 913-916.

MacLennan AH (2011). HRT in difficult circumstances: are there any absolute contraindications? Climacteric 14: 409-417.

Meirow D, Hardan I, Dor J, Fridman E, et al. (2008). Searching for evidence of disease and malignant cell contamination in ovarian tissue stored from hematologic cancer patients. Hum. Reprod. 23: 1007-1013.

Michelsen TM, Pripp AH, Tonstad S, Trope CG, et al. (2009). Metabolic syndrome after risk-reducing salpingooophorectomy in women at high risk for hereditary breast ovarian cancer: a controlled observational study. Eur. $J$. Cancer 45: 82-89.

Miller DM, Ehlen TG and Saleh EA (1997). Successful term pregnancy following conservative debulking surgery for a stage IIIA serous low-malignant-potential tumor of the ovary: a case report. Gynecol. Oncol. 66: 535-538.

Miller K, Price JH, Dobbs SP, McClelland RH, et al. (2008). An immunohistochemical and morphological analysis of post-chemotherapy ovarian carcinoma. J. Clin. Pathol. 61: 652-657.

Rahma OE, Ashtar E, Czystowska M, Szajnik ME, et al. (2012). A gynecologic oncology group phase II trial of two p53 peptide vaccine approaches: subcutaneous injection and intravenous pulsed dendritic cells in high recurrence risk ovarian cancer patients. Cancer Immunol. Immunother. 61: 373-384.

Romero I and Bast RC Jr. (2012). Minireview: human ovarian cancer: biology, current management, and paths to personalizing therapy. Endocrinology 153: 1593-1602.

Rosendahl M, Andersen CY, Ernst E, Westergaard LG, et al. (2008). Ovarian function after removal of an entire ovary for cryopreservation of pieces of cortex prior to gonadotoxic treatment: a follow-up study. Hum. Reprod. 23: 2475-2483.

Takami H, Sentani K, Matsuda M, Oue N, et al. (2012). Cytokeratin expression profiling in gastric carcinoma: clinicopathologic significance and comparison with tumor-associated molecules. Pathobiology 79: 154-161. 